\title{
A Phytochrome-Derived Photoswitch for Intracellular Transport
}

\author{
Max Adrian, ${ }^{\circledR}$ Wilco Nijenhuis, Rein I. Hoogstraaten, Jelmer Willems, and Lukas C. Kapitein*(i) \\ Cell Biology, Department of Biology, Faculty of Science, Utrecht University, $3584 \mathrm{CH}$ Utrecht, The Netherlands \\ Supporting Information
}

ABSTRACT: Cells depend on the proper positioning of their organelles, suggesting that active manipulation of organelle positions can be used to explore spatial cell biology and to restore cellular defects caused by organelle misplacement. Recently, bluelight dependent recruitment of specific motors to selected organelles has been shown to alter organelle motility and positioning, but these approaches lack rapid and active reversibility. The light-dependent interaction of phytochrome B with its interacting factors has been shown to function as a photoswitch, dimerizing under red light and dissociating under far-red light. Here we engineer phytochrome domains into photoswitches for intracellular transport that enable the reversible interaction between organelles and motor proteins. Using patterned illumination and

Orthogonal photoswitches for intracellular transport

Endosomes

Peroxisomes

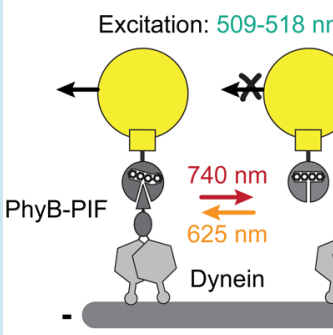

Microtubule

live-cell imaging, we demonstrate that this system provides unprecedented spatiotemporal control. We also demonstrate that it can be used in combination with a blue-light dependent system to independently control the positioning of two different organelles. Precise optogenetic control of organelle motility and positioning will provide a better understanding of and control over the spatial biology of cells.

KEYWORDS: cell biology, optogenetics, phytochrome B, organelles, live-cell imaging

Tn many cell types the proper subcellular positioning of organelles is crucial for correct functioning and also determines their inheritance in daughter cells after cell division. ${ }^{1,2}$ Even in cells with a less defined cellular architecture, many organelles rely on contacts with other organelles for functions like metabolism, lipid transfer and signaling. ${ }^{3-6}$ Despite this importance, studying the role of organelle positioning has until recently been difficult due to the lack of adequate tools to position organelles at will in living cells. ${ }^{1}$ We have previously shown that cellular cargoes such as organelles can be positioned in living cells by chemically induced recruitment of constitutively active motor proteins. ${ }^{7}$ Recently, we and others have extended this toolbox by making this heterodimerization light-inducible, ${ }^{8,9}$ allowing to control organelle positioning with subcellular precision or in vivo. ${ }^{10}$

In these light-inducible approaches, dimerization of LOV domains $^{11}$ or cryptochromes ${ }^{12}$ is induced by blue light. Alternatively, UV light can be used to uncage a chemical dimerizer, but only at the expense of spatial precision and repeated reversibility. ${ }^{13}$ Nevertheless, also the use of blue lightsensitive domains severely limits the design of live imaging experiments, because GFP-tagged proteins cannot be imaged without activation of the dimerization system. In addition, these dimerization systems rely on their intrinsic dissociation kinetics for unbinding of the motors after returning to dark conditions. Even though binding affinities and rates of these systems have been optimized to suit a variety of applications, ${ }^{11,14,15}$ a direct "off-switch" that actively dissociates motors from their cargoes should enable more precise positioning. Finally, an orthogonal heterodimerization system that responds to a different color would allow multicolor control of the relative positioning of different organelles.

Light-inducible dimerization domains in phytochromes from Arabidopsis thaliana have been shown to behave as bidirectional photoswitches. ${ }^{16}$ The amino-terminal domain of phytochrome $\mathrm{B}$ (PhyB) can be switched from a red light-absorbing Pr state to a far-red light-absorbing Pfr state with red light in the presence of the cofactor phycocyanobilin (PCB). In the Pfr state, PhyB interacts with Phytochrome Interacting Factors (PIF) ${ }^{17}$ and importantly, remains bound until illuminated with far-red light, which reverts $\mathrm{PhyB}$ into its $\operatorname{Pr}$ state and thereby dissociates from PIF rapidly. ${ }^{16}$ This photoswitchable system has so far been used to regulate gene transcription in yeast, mammalian cells and in vivo in zebrafish ${ }^{18-20}$ or to engineer light-sensitive signal cascades. ${ }^{16,21}$ Here, we develop organelle positioning assays using phytochrome-derived dimerization domains to establish switch-like control over organelle movement on subcellular scale with high temporal resolution using red and far-red light.

\section{RESULTS AND DISCUSSION}

To couple motor domains to organelles, we engineered fusion constructs of PhyB(1-908) and GFP-labeled organelle markers and fused PIF6 $(1-100)$ to previously characterized motor

Received: November 5, 2016

Published: March 24, 2017 


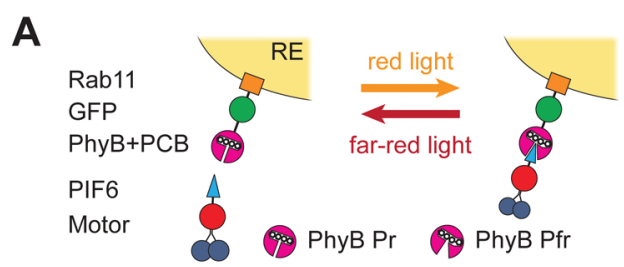

B
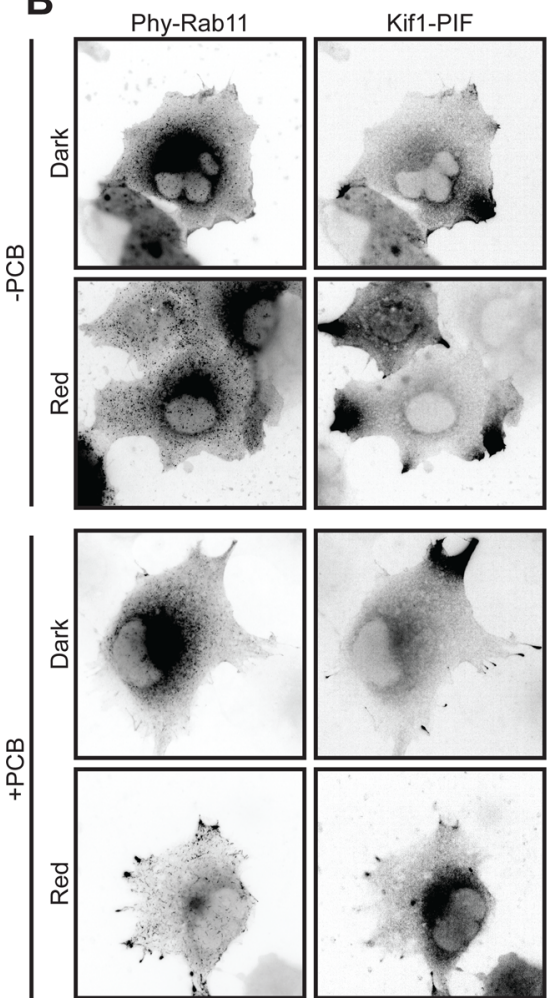
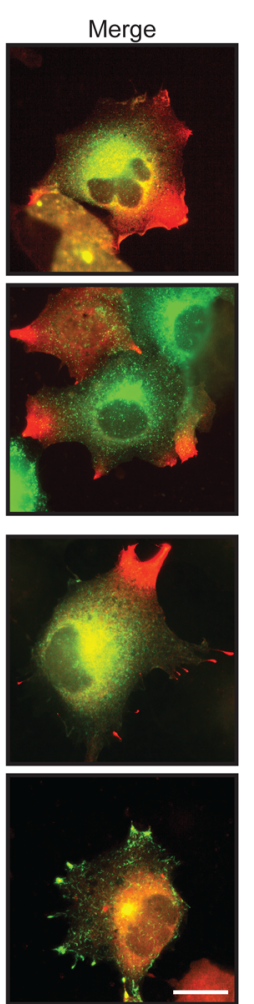

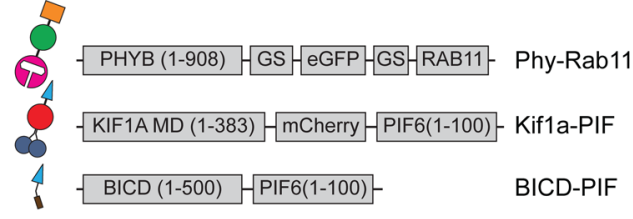

C
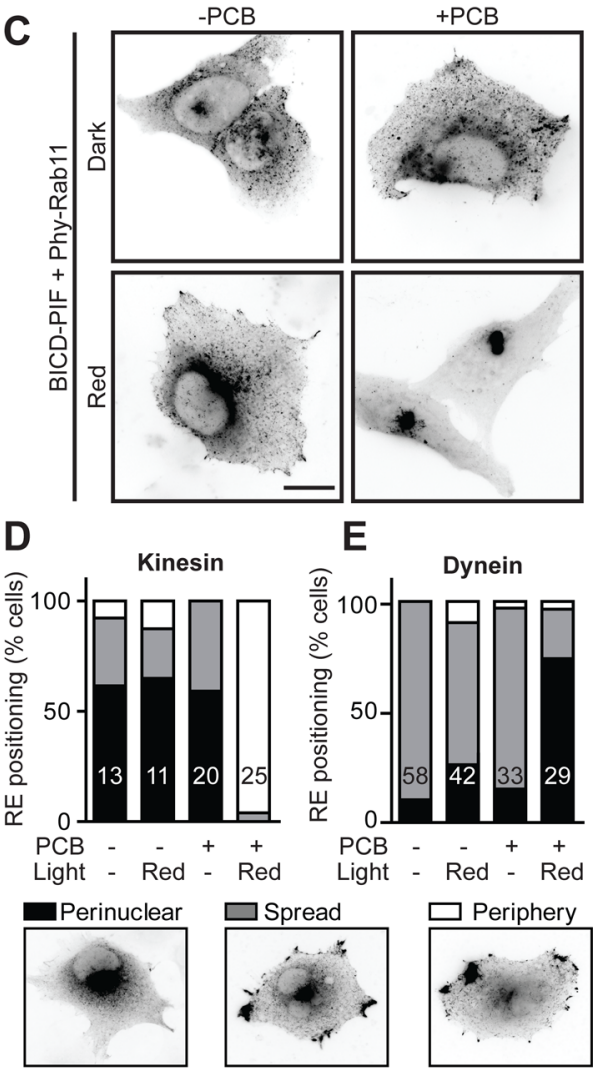

Figure 1. Repositioning of recycling endosomes using light-induced phytochrome-based dimerization with motor proteins. (A) Assay and constructs. Recycling endosomes were tagged with fluorescently labeled Rab11 fused to PhyB(1-908) (Phy-Rab11). Upon exposure to red light, PhyB changes confirmation from the Pr to the Pfr state, allowing it to bind PIF6 which is coupled to Kif5b(1-807)-mCherry (Kif5-PIF), Kif1a(1-383)-mCherry (Kif1-PIF), or the dynein adaptor BICD(1-500) (BICD-PIF). (B) Distribution of recycling endosomes tagged with Phy-Rab11 in COS-7 cells expressing Kif1-PIF with or without PCB incubated either in the dark or under red light for $30 \mathrm{~min}$. (C) Distribution of recycling endosomes tagged with Phy-Rab11 in U2OS cells expressing (color-less) BICD-PIF with or without incubation of PCB either in the dark or under red light for 30 min. (D,E) Quantification of manually scored Phy-Rab11 localization of experiments shown in (B) and (C), respectively. Numbers in bars indicate $n$ cells analyzed for each condition. Scale bars are $20 \mu \mathrm{m}$.

domains of kinesin and dynein motors. ${ }^{7,9}$ Light-induced dimerization should then induce transport of the target cargo to the cell periphery or the microtubule organizing center (MTOC), respectively (Figure 1A). First this system was tested in fixed cells that were globally exposed to red light for $30 \mathrm{~min}$ prior to fixation. Recycling endosomes tagged with Phy-Rab11 were spread throughout the cytoplasm and partly enriched in the perinuclear region in control conditions. In the presence of kinesin-3 derived Kif1-PIF and PCB, repositioning of recycling endosomes could be induced with red light illumination in $96 \%$ of the cells imaged (Figure 1B,D). Moreover, to test the universality of this system, we repeated the experiment with the motor domain of Kif5b, a member of the kinesin-1 family. Recruitment of Kif5-PIF to recycling endosomes also induced their robust repositioning to the cell periphery (Supporting Information Figure S1). Similarly, by recruiting BICD-PIF to recycling endosomes, dynein-mediated transport to the MTOC was induced and recycling endosomes heavily accumulated at the MTOC in $74 \%$ of cells (Figure $1 \mathrm{C}, \mathrm{E}$ ).
In addition, we performed the same kinesin recruitment assays with peroxisomes labeled with Phy-Pex. Both coupling of kinesin-1 or kinesin-3 motor domains resulted in efficient repositioning of peroxisomes to the cell periphery after illumination with red light in $96 \%$ and $88 \%$ of cells imaged, respectively (Supporting Information Figure S2). Together these results demonstrate that our strategy to reposition cellular organelles by recruiting motor proteins using phytochromederived dimerization domains can efficiently be used for different combinations of organelles and motors.

To further optimize organelle tagging and to reduce the construct size of the fusion protein, a shorter amino-terminal truncation of $\mathrm{PhyB}^{20}$ was tested. However, already in the absence of light, significant repositioning of endosomes to the periphery was observed after addition of PCB in $70 \%$ and $44 \%$ of cells expressing Phy(1-650)-Rab11 and Kif5-PIF or Kif1PIF, respectively. Moreover, the coupling was irreversible under far-red light (Supporting Information Figure S3). Such irreversible hyperactivity of short amino-terminal truncations 

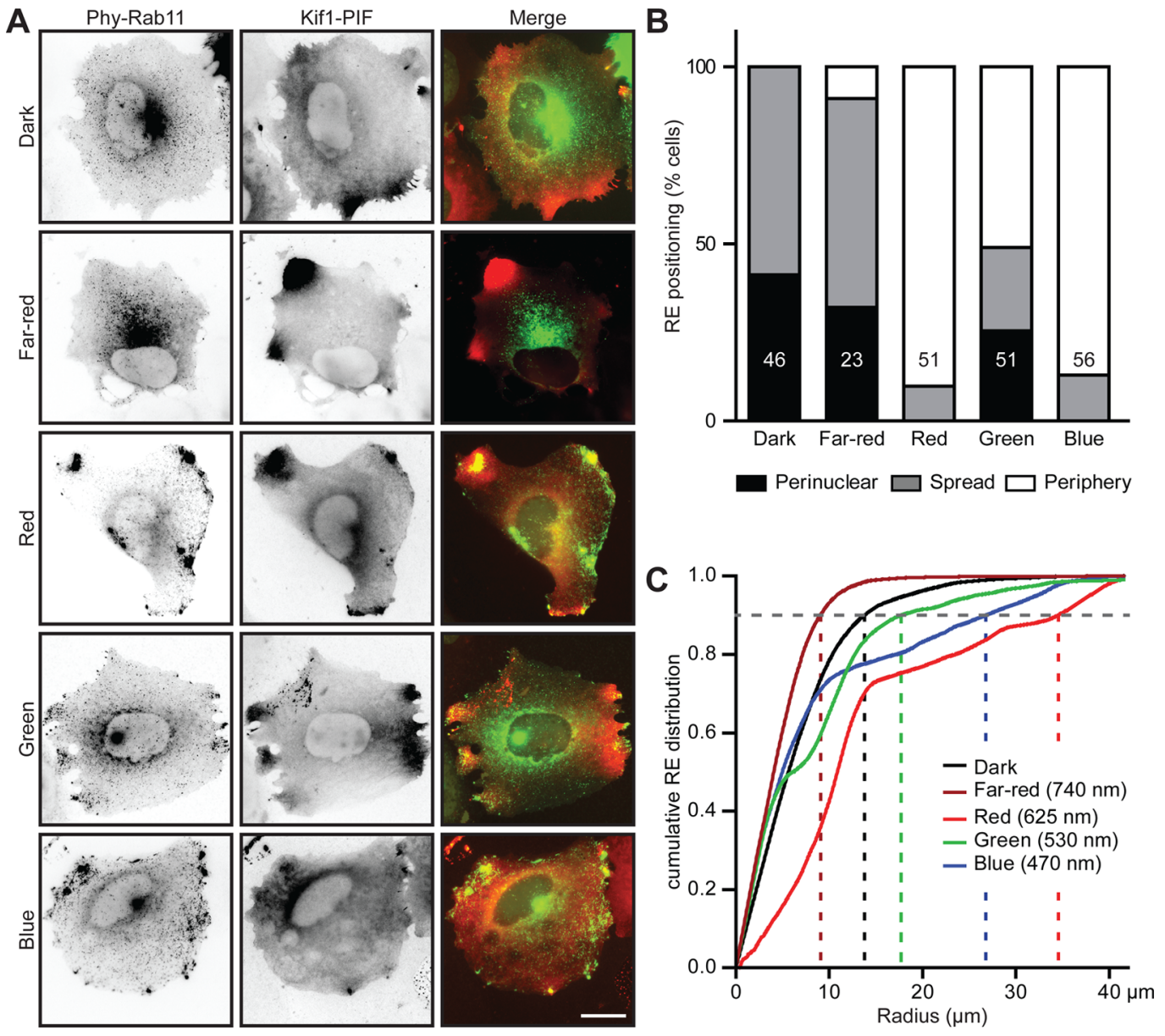

Figure 2. PhyB-PIF interaction is sensitive to a range of visible wavelengths and requires far-red illumination to dissociate. (A) Distribution of recycling endosomes tagged with Phy-Rab11 in U2OS cells expressing Kif1-PIF incubated with PCB either in the dark or under indicated colored light for $30 \mathrm{~min}$. (Red: $625 \mathrm{~nm}$, Green: $530 \mathrm{~nm}$, Blue: $470 \mathrm{~nm}$, all $\sim 20 \mu \mathrm{W} / \mathrm{cm}^{2}$, Far-red: $740 \mathrm{~nm}, \sim 400 \mu \mathrm{W} / \mathrm{cm}^{2}$ ). (B) Quantification of manually scored Phy-Rab11 localization of experiments shown in (A). Numbers in bars indicate $n$ cells analyzed for each condition. (C) Cumulative measurements of Phy-Rab11 signal measured radially from the cell center to the periphery for cells shown in (A). Intersection with horizontal gray line indicates radius encompassing $90 \%$ of all Phy-Rab11 signal. Scale bar is $20 \mu \mathrm{m}$.

has also been reported previously ${ }^{16,22}$ and we therefore abandoned this approach.

To directly assess repositioning dynamics, we next carried out live-cell imaging experiments. Since exposure of PhyB to low levels of ambient light may already activate PIF binding, ${ }^{22}$ addition of PCB was delayed until briefly before start of illumination. This allowed better handling of samples and prevented unintentional premature activation. Yet, we consistently noticed that the blue light used to excite GFP was already sufficient to activate recycling endosome repositioning in our assays. Therefore, we tested the reactivity of recycling endosome repositioning in a fixed experiment in which cells were either not illuminated or illuminated with far-red, red, green or blue light before fixation (Figure 2A). Indeed, exposure to blue light resulted in repositioning of recycling endosomes to the cell periphery with similar efficiency as red light illumination in $87 \%$ and $90 \%$ of cells, respectively. Similarly, $51 \%$ of the cells illuminated with green light showed endosome accumulations at the cell periphery, indicating that this motor recruitment assay is very sensitive to activation by light of the entire visible spectrum (Figure 2B). As expected, the relative radial distribution of recycling endosomes in far-red light illuminated cells was smallest, with $90 \%$ of endosomal fluorescence located in $9 \mu \mathrm{m}$ distance from the cell center, compared to $14,18,27$ and $35 \mu \mathrm{m}$ for dark, green, blue and red illuminated cells of similar size, respectively (Figure 2C). Thus, while organelle repositioning is not exclusively activated by red light, it can be prevented by far-red illumination, suggesting that the system may still be used for effective on/off photoswitching of organelle motility.

During subsequent live-imaging experiments, cells were exposed to far-red light before the start of the acquisition or whenever motors should be dissociated from recycling endosomes (Figure 3A). During illumination with $625 \mathrm{~nm}$ light repositioning of recycling endosomes to the cell periphery could be followed (Figure 3B, Movie S1) and quantified (Figure $3 \mathrm{C}-\mathrm{E}$ ). The increase in peripheral Phy-Rab11 fluorescence intensity was accompanied by a simultaneous loss of intensity in the perinuclear region of the cell (Figure 3C). The frame-to-frame correlation index, which quantifies overall motility by comparing the overlap between subsequent frames ( 1 indicates completely similarity, 0 means completely dissimilarity), ${ }^{7,9}$ decreased nearly instantly after onset of 625 $\mathrm{nm}$ illumination, indicating that motility of recycling endosomes increased instantly after onset of $625 \mathrm{~nm}$ illumination(Figure 3E). The peripheral intensity and displacement radius (Figure 3C,D) of recycling endosomes increased shortly after as endosomes move toward the cell periphery. The correlation 


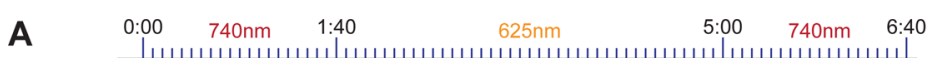

B

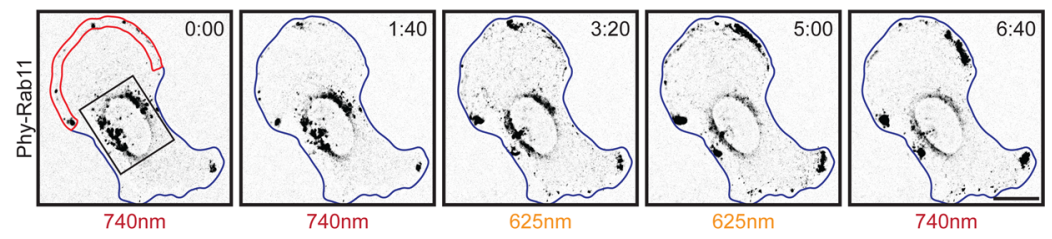

C

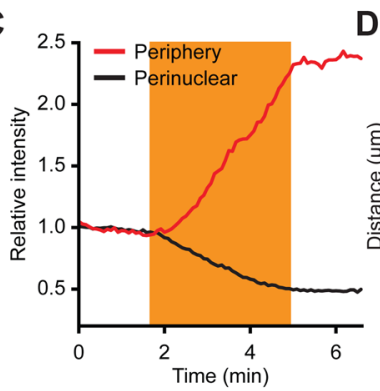

D E

$\mathbf{F}$
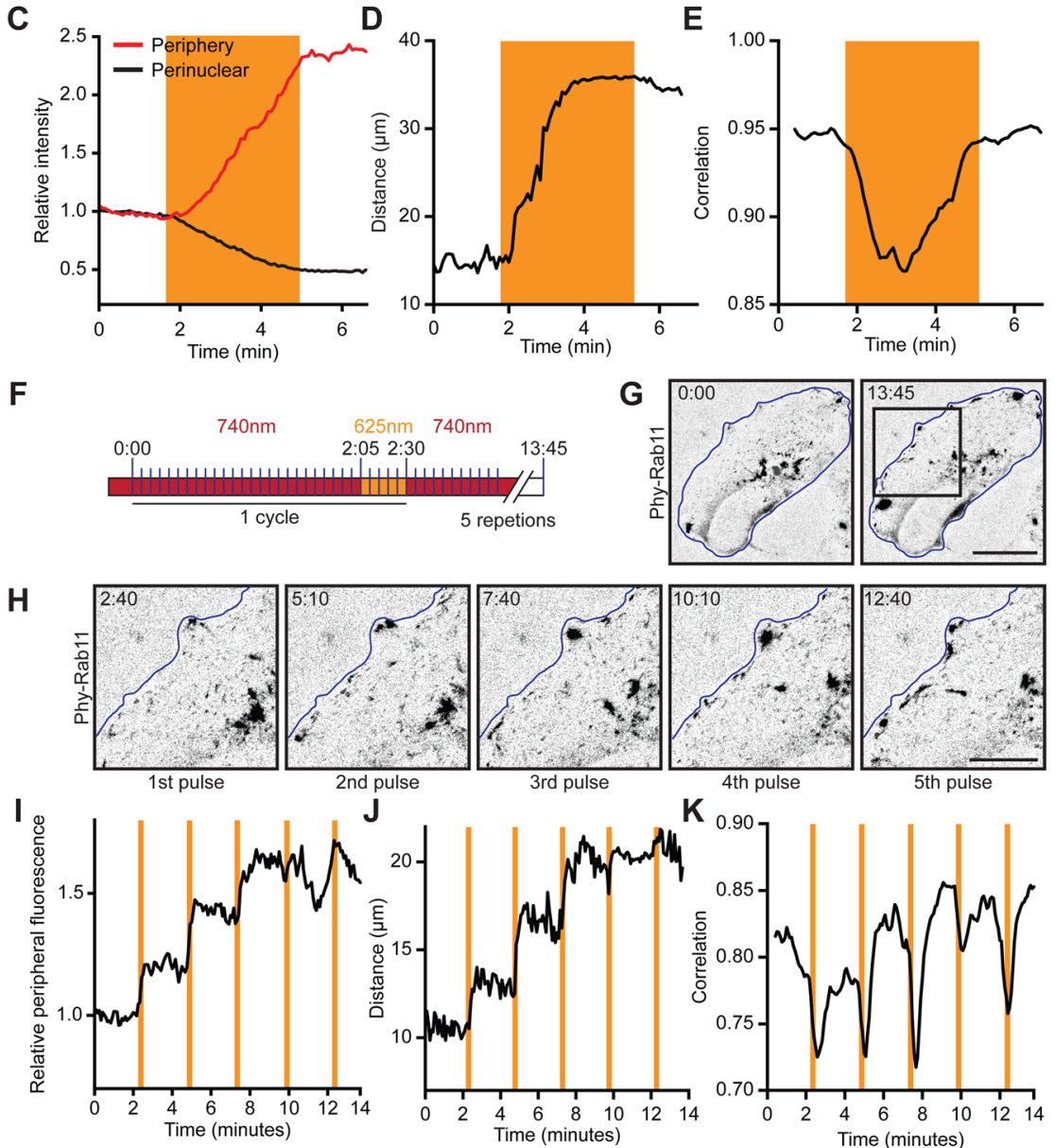

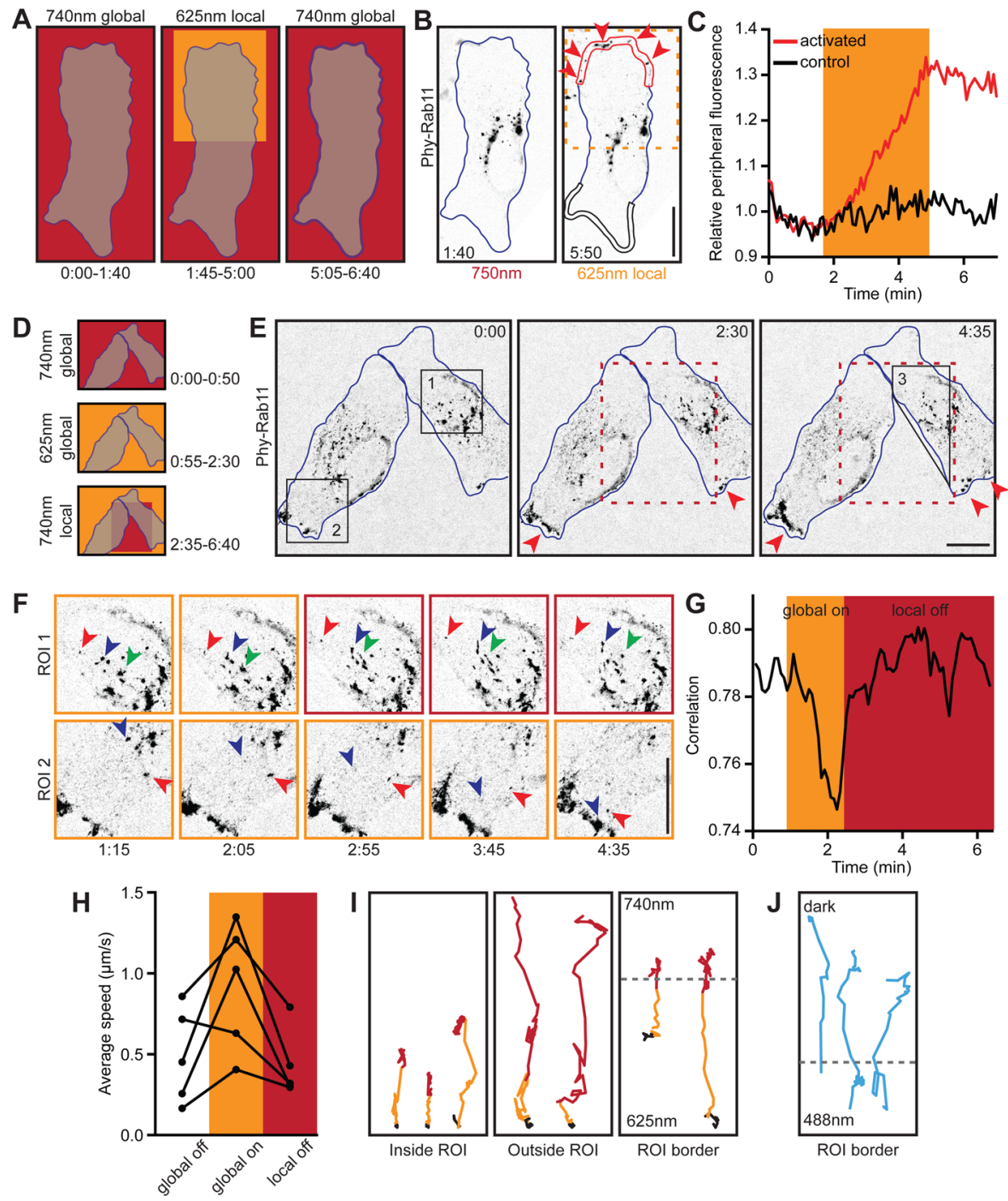

Figure 4. Local illumination with patterns of red/far-red light allows spatial control over organelle positioning. (A) Schematic representation of light exposure during live-cell imaging experiment. After global illumination with $740 \mathrm{~nm}$ light for 20 frames, a small area is illuminated with $625 \mathrm{~nm}$ light for 40 frames, followed by global $740 \mathrm{~nm}$ light to dissociate all motors. Illuminations were interspersed with GFP imaging excitation light every $5 \mathrm{~s}$. Blue lines indicate cell outlines. Orange and red boxes indicate 625 and $740 \mathrm{~nm}$ illumination, respectively. (B) Recycling endosome localization in U2OS cells before and after local dimerization to Kif1-PIF with $625 \mathrm{~nm}$ light in region indicated with orange dashed box. See also Movie S3. (C) Quantification of peripheral Phy-Rab11 at $625 \mathrm{~nm}$ illuminated region (red) and control region (black) as indicated in (B). (D) Schematic representation of light exposure during live-cell imaging experiment. After global activation with $625 \mathrm{~nm}$ light for 20 frames, a small area is illuminated with $740 \mathrm{~nm}$ light for 50 frames to locally dissociate motors. (E) Recycling endosome localization during local dissociation of Phy-Rab11 from Kif5-PIF as outlined in (D). Red dashed box indicates $750 \mathrm{~nm}$ illuminated area. See also Movie S4. (F) Zooms of regions 1 and 2 shown in (E). Colored arrowheads highlight position of the same recycling endosomes in consecutive frames. Color of image border indicates wavelength of local illumination. (G) Frame-to-frame correlation (from 0 to 1) indicating motility of Phy-Rab11 in region 3 shown in (E). (H) Average speed of individual recycling endosomes in the area illuminated with $740 \mathrm{~nm}$ light (red dashes in (E)). (I) Trajectories of recycling endosomes inside, outside or on the border of locally deactivated area. Trajectories are shown in black during initial global $740 \mathrm{~nm}$ illumination (frame 1-10), orange during global $625 \mathrm{~nm}$ illumination (frames 11-30) and red during local $740 \mathrm{~nm}$ illumination (frames $31-80$ ). Note that these time intervals are not equally long. (J) Trajectories of recycling endosomes locally activated using the blue-light sensitive TULIP system in Cos7 cells and remaining mobile in the nonilluminated area. Scale bars are $30 \mu \mathrm{m}$.

photoswitch to increase temporal precision of organelle repositioning.

In addition to increased temporal resolution when using the phytochrome system, patterned illumination with red and farred light should allow local activation/deactivation of organelle repositioning at subcellular resolution. To test this, we actively dissociated motors from recycling endosomes with global farred illumination, while a smaller region of the cell was illuminated with red light to locally induce organelle repositioning (Figure 4A). Peripheral accumulation of recycling endosomes was observed in the region that was illuminated with red light, whereas the far-red illumination prevented repositioning elsewhere (Figure 4B,C, Movie S3). To test whether organelle repositioning induced with red light could also locally be stopped using far-red light, we adjusted the illumination scheme to illuminate a subcellular region with farred light after prior global activation of repositioning using red light (Figure 4D). Recycling endosomes initially started moving toward the cell periphery, but were prevented from reaching the cell periphery in far-red illuminated areas (Figure 4E,F, 
Movie S4). This local inactivation of organelle repositioning could further be quantified by correlation analysis, showing decreased motility in a far-red illuminated area (Figure 4G). In addition, measuring the average speed of recycling endosomes in this area showed that the red light-induced increase was reversed after local deactivation with far-red light (Figure 4H). Single trajectories from recycling endosomes show that endosome motility in far-red illuminated areas rapidly decreased while endosomes outside this region remained mobile. Interestingly, endosomes crossing into the far-red illuminated area also quickly stopped moving, because the farred light actively reversed the interaction between motors and organelles (Figure 4I). Thus, phytochrome-mediated heterodimerization combined with spatially patterned illumination allows controlling organelle dynamics with high spatiotemporal precision.

We next compared the PhyB-PIF system with the blue-light sensitive TULIP system that we previously used. The bidirectional control of heterodimerization using red and farred light provides two modes of control that are not available with the blue-light based system: acute inhibition and sustained activation. Indeed, whereas we observed a rapid arrest of phytochrome-controlled motility when organelles moved from red to far-red illuminated areas (Figure 4I), such acute inhibition of motility was not observed using blue-light dependent heterodimerization and local illumination, because the slow reversal time scales of 30-60 s and motor velocities of $1-2 \mu \mathrm{m} / \mathrm{s}$ resulted in long excursions into the nonilluminated area (Figure $4 \mathrm{~J}$ ). In addition, when recycling endosomes were relocalized to the cell periphery using a blue-light based system, these organelles spontaneously regained their original distribution when left in the dark for half an hour (Figure 5A). In contrast, when using the PhyB-PIF system, endosomes were still enriched at the periphery after half an hour in the dark (Figure 5B). These results demonstrate that the two-color bidirectional control provide by the PhyB-PIF system adds unique capabilities to the organelle repositioning toolbox.

Finally, we tested whether we could combine both systems to achieve independent control over the positioning of different organelles in one experiment. Recycling endosomes were labeled with PhyB fused to the yellow fluorescent protein Venus, which could be excited with 509-518 nm light without activating TULIP-based heterodimerization, whereas unwanted activation of PhyB could be reversed used far-red light (Figure 5C). Peroxisomes were labeled with the light-sensitive part of the TULIP system (LOVpep) fused to mRFP. At the start of the experiment, Venus and RFP could be excited with green and red light, while PhyB activation was prevented using far-red light. Subsequent PhyB activation with red light resulted in dynein-driven perinuclear enrichment of recycling endosomes, whereas peroxisome positioning was unaffected until illumination with blue light induced kinesin-dependent transport that moved the peroxisomes out of the perinuclear region (Figure $5 \mathrm{D}-\mathrm{F})$. Thus, by carefully choosing fluorophores and illumination strategy, blue-light controlled and red-light controlled systems can be used independently in a single cell and also combined with two-color imaging.

In conclusion, we demonstrate that phytochrome-derived motor-cargo switches allow fast and actively reversible coupling of organelles and motor proteins. This interaction could be spatiotemporally controlled by exposing regions of interest to red and far-red light to induce or reverse binding, respectively. The main advantage over blue light-inducible recruitment of
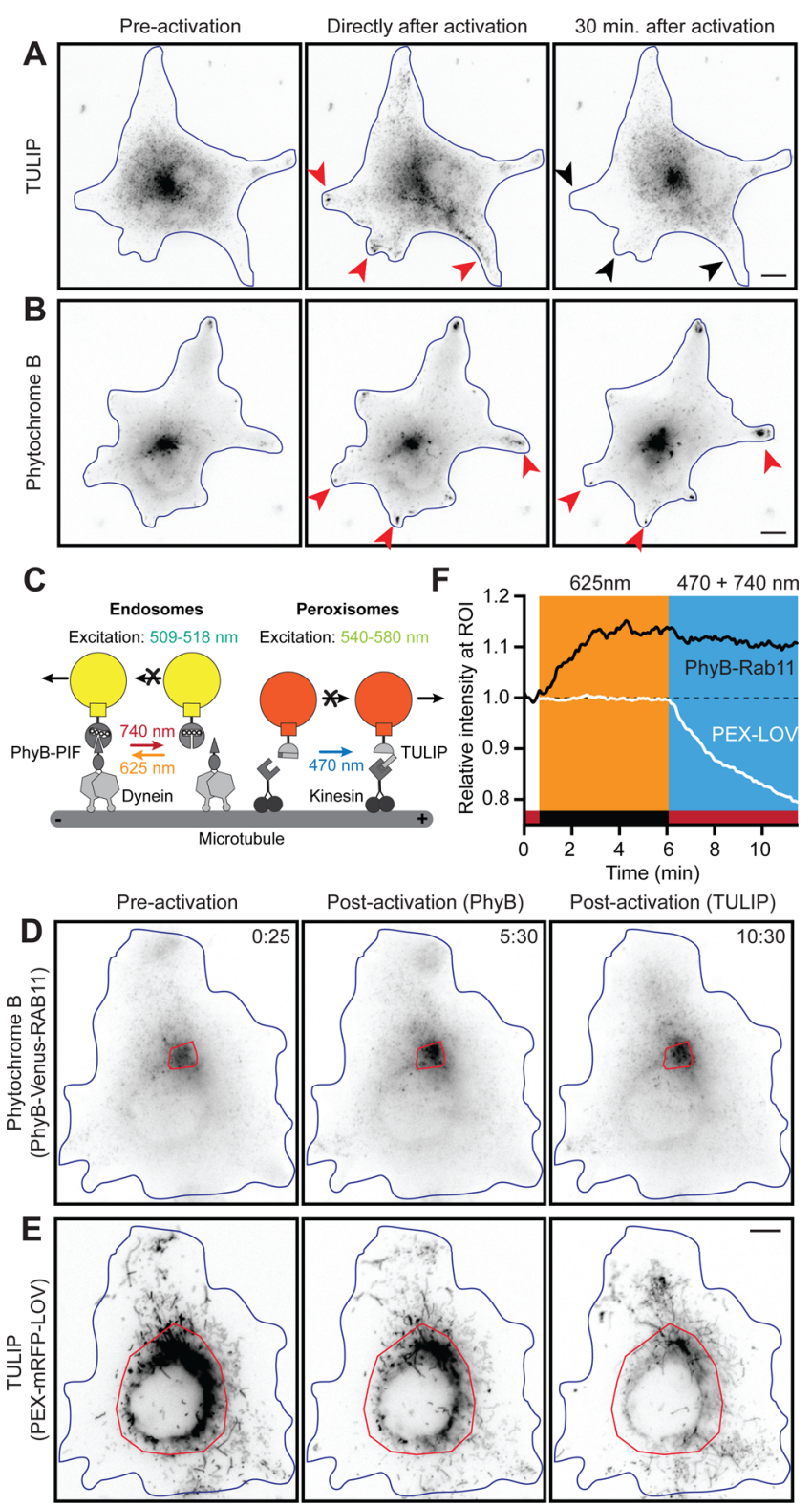

Figure 5. Combining blue-light sensitive and red-light sensitive systems for orthogonal control of the transport of different organelles. (A) Recycling endosome localization before, directly after and $30 \mathrm{~min}$ after global association of LOV-coupled Rab11 with Kif5-ePDZb1 in COS-7 cells. Blue lines indicate cell outlines. (B) Recycling endosome before, directly after and $30 \mathrm{~min}$ after global association of Phy-Rab11 with Kif17-PIF in Cos-7 cells. Excitation and illumination light was paused for $30 \mathrm{~min}$ following activation of PhyB-PIF dimerization to prevent reassociation by the excitation light. (C) Schematic illustrating the different illumination wavelengths used for excitation and activation required to combined two-color imaging with both the PhyB-PIF system and the TULIP system. (D,E) Localization of recycling endosomes (D) and peroxisomes (E) in a single COS-7 cell, before activation of either the TULIP or Phytochrome B systems (left panels), after induced dimerization of PhyB-Venus-Rab11 with BICDPIF6 (middle panels), and after subsequent induced dimerization of PEX-mRFP-LOV with Kif1A-ePDZb1 (right panels). Blue lines indicate cell outlines, all times given in min:s. Scale bar: $10 \mu \mathrm{m}$. (F) Measurements of fluorescence intensity of PhyB-Rab11 (black) near the microtubule organizing center (red region in D) and of PEX-LOV (white) in the perinuclear region (red region in E), showing orthogonal activation of the TULIP and PhyB-PIF systems in a single cell. Scale bars: $10 \mu \mathrm{m}$. 
motors to organelles lies in the fast and active reversibility of the phytochrome system with a separate wavelength. ${ }^{16}$ Even though we found that phytochromes are sensitive to a broad spectrum, including blue light, far-red illumination actively dissociates PhyB-PIF interactions during live imaging of GFPlabeled structures. In addition, we demonstrated that the phytochrome system can be combined with blue light-sensitive systems to allow orthogonal control of positioning of two organelles, similar to previously demonstrated gene expression assays. $^{23}$

Dependence on PCB as a cofactor could be considered a limiting factor for this system, because it is not expressed in animal cells. However, for our purposes, this dependence enabled us to easily handle transfected cells in ambient light conditions before the start of the experiments, because addition of purified PCB to the cell medium for $1 \mathrm{~h}$ was sufficient. In other model systems, particularly in vivo, this may prove more challenging. However, injection of PCB into zebrafish embryos has been reported ${ }^{19}$ and cultured cells have also been engineered to metabolize abundant biliverdin into $\mathrm{PCB}^{24}$ to overcome this limitation. Recently, a photoswitchable dimerization system has been reported that uses biliverdin in its chromophore and is activated by far-red light. ${ }^{25}$ This system may thus overcome the requirement of exogenous compounds and the broad activation spectrum reported here, but its photoswitch behavior might be less effective as reversal by redlight was not complete. We expect that the assays reported here, as well as future improvements of the optogenetic dimerization systems, will contribute to a better understanding of the spatial biology of cells by enabling precise control of organelle positioning in dynamic cells and tissues as well as in well-controlled minimal cell reconstitutions.

\section{METHODS}

DNA Constructs. The following constructs have been described: PEX3-mRFP-LOVpep, FRB-TagBFP-LOVpep, FKBP-tagRFPt-Rab11, Kif1A(1-383)-GFP-ePDZb1, Kif5B(1-807)-GFP-ePDZb1 ${ }^{9}$ and Kif17md-GFP-FRB. ${ }^{26}$ To produce $\mathrm{p} \beta$-actin Kif1A-, Kif5B-, Kif17-, and BICDN-mCherryPIF6, previously characterized motor-domain fusion proteins ${ }^{9,26}$ were digested with NotI and SalI and ligated with PCR amplified mCherry-PIF6 (5'-TTTTGTCGACTATGGTGAGCAAGGGCGAG- $3^{\prime}$ and $5^{\prime}$-TTTGCGGCCGCTTAGTCAACATGTTTATTGCTTTCCAACATGT-3'), generated from pSV40-mYFP-PIF6APB ${ }^{16}$ (addgene plasmid 22276) (5'-TTTAGGCGCGCCTATGATGTTCTTACCAACCGATTATTGTT-3' and 5'-ATTTGTCGACTTAGTCAACATGTTTATTGCTTTCCAACATGT-3').

PhyB(1-908)-eGFP-GS-Rab11 was generated from PCR amplified eGFP-Rab11 containing a 29 amino acid long GS linker separated by NheI and SnaBI sites (5'-TTTGAATTCGGTGGCGGGGGCAG- $3^{\prime}$ and $5^{\prime}$-TTTGCGGCCGCTTAGATGTTCTGACAGCACTGCACC-3') inserted into PhyB-mCHERRY-CAAX ${ }^{16}$ (addgene plasmid 22282) using EcoRI and NotI sites. PhyB(1-908)-Venus-GS-Rab11 was generated from PCR amplified Venus flanked by EcoRI and NheI sites (5'-AGCGAATTCGGTGGCGGGGGGAGCGGGGGTGGGAGTAAGCTTGTGAGCAAGGGCGAGGAG$3^{\prime}$ and 5-'AACGGCTAGCCTTGTACAGCTCGTCCATGCCG-3') inserted into PhyB(1-908)-eGFP-GS-Rab11.

Similarly, PHYB(1-650)-eGFP-GS-Rab11 was generated by Gibson cloning of PCR amplified eGFP-Rab11 (5'-GTTAGGTTCTAGAGGTGGCGGGGGCA- ${ }^{\prime}$ and $5^{\prime}$-CTCGAGA-
AGTACTAGTTTAGATGTTCTGACAGCACTGCACCT$\left.3^{\prime}\right)$ and PhyB (5'-AAGCTTGCCACCATGGTTTCCGGAGTCGGG-3' and 5'- CCTCTAGAACCTAACTCATCAATCCCCTGTTCC- $\left.3^{\prime}\right)$ into NcoI and BcuI sites of $\mathrm{p} \beta$-actin. PCR amplified human PEX26 (246-305) with NheI/NotI sites (5'TTTGCTAGCACGCACTTCTTTTCTCTGCCC-3' and $5^{\prime}$ GCGGCCGCTTCAGTCATG-3') or with SnaBI sites (5'ATGCTACGTAACGCACTTCTTTTCTCTGCCC-3' and 5'-ATGCTACGTATCAGTCATGGATGCGGAGCT-3') was ligated into PhyB(1-908)-eGFP-GS-Rab11 to generate PhyB(1-908)-eGFP-PEX26 or PhyB(1-650)-eGFP-GSRab11 to generate PhyB(1-650)-eGFP-GS-PEX26, respectively.

Cell Culture and Fluorescence Microscopy. African green monkey kidney fibroblast-like COS-7 (Figures 1B, 3J, 5, S1, S2) and human bone osteosarcoma U2OS (Figures 1C, 2, 3, 4, S3) cells were maintained in Dulbecco's modified Eagle's medium (DMEM) supplemented with $10 \%$ fetal calf serum and penicillin/streptomycin at $37{ }^{\circ} \mathrm{C}$ in $5 \% \mathrm{CO}_{2}$.

For fixed experiments, cells were seeded on glass coverslips and transfected with $1.8 \mu \mathrm{g}$ plasmid DNA (1:2 ratio cargo:motor) and polyethylenimine (PEI) (1:3) for 16-24 h prior to fixation. Cells were incubated with $15 \mu \mathrm{M}$ phycocyanobilin (PCB, Frontier Scientific, batch FSI154496) for $1 \mathrm{~h}$ and exposed with light illumination schemes described below, followed by fixation with $4 \%$ paraformaldehyde for $10 \mathrm{~min}$ at room temperature in the dark, washing with PBS and mounting on glass slides using Vectashield or Mowiol. Epifluorescence images were acquired using a 40× (Plan Fluor, NA 1.3, Nikon) or 100× (Plan Apo Lambda, NA 1.45, Nikon) oil-immersion objective on a Nikon Eclipse $\mathrm{Ni}$ upright fluorescence microscope equipped with a Coolsnap HQ2 CCD camera (Photometrics), a mercury lamp (Osram) and emission filters ET-GFP (49002), ET-mCherry (49008) (both Chroma) and controlled with NIS software (Nikon). For live cell imaging, cells were transfected with Fugene6 (Promega) and imaged 16-24 h later after incubation with $15 \mu \mathrm{m}$ PCB for at least $1 \mathrm{~h}$ in fresh culture medium at $37^{\circ} \mathrm{C}$ and $5 \% \mathrm{CO}_{2}$. For live-cell imaging of Rab11 using the TULIP system, $100 \mathrm{nM}$ of rapalog (AP21967, Ariad Pharmaceuticals) was added at least 20 min prior to imaging in order to couple FRB-LOV to FKBPRab11. Cells were imaged with a 40× (Plan Fluor, NA 1.3, Nikon) oil-immersion objective on a Nikon $\mathrm{Ti}$ inverted microscope equipped with a sample incubator (Tokai-Hit), a mercury lamp (Osram), ET-GFP (49002), ET-mCherry (49008) and ET 514 nm Laser Bandpass (49905) filter cubes (all Chroma) and a Coolsnap HQ2 CCD camera (Photometrics), controlled with $\mu$ Manager 1.4 software.

Illumination Schemes. For fixed experiments, transfected cells were incubated in the dark or in red, blue or green light using either a custom array of RGB-LEDs $(625 \mathrm{~nm}, 530 \mathrm{~nm}$, $\left.470 \mathrm{~nm}, \sim 20 \mu \mathrm{W} / \mathrm{cm}^{2}\right)$, custom far-red LEDs $(740 \mathrm{~nm}, \sim 400$ $\left.\mu \mathrm{W} / \mathrm{cm}^{2}\right)$ or a commercial color adjustable LED light $(\sim 40$ $\mu \mathrm{W} / \mathrm{cm}^{2}$, LivingColors 70019, Philips) for $30 \mathrm{~min}$.

For live-cell imaging using the Phytochrome B system, cells were illuminated with $>725 \mathrm{~nm}$ light prior to acquisition using the halogen transmission light path and a 725 cut-on colored glass filter (FSQ-RG9, Newport) at $\sim 10 \mathrm{~mW} / \mathrm{cm}^{2}$ on the specimen. To illuminate the cells during acquisition we used a Polygon 2000 digital mirror device (DMD) equipped with 470, 625, and $740 \mathrm{~nm}$ LEDs (all Mightex) that exposed ROIs between imaging frames with up to $300 \mathrm{~mW} / \mathrm{cm}^{2}, 70 \mathrm{~mW} / \mathrm{cm}^{2}$ and $30 \mathrm{~mW} / \mathrm{cm}^{2}$, respectively (powers measured at the back 
focal plane and converted to intensity using the projected area of the DMD). Light exposure was synchronized with camera frames using camera-evoked TTL triggers. For global illumination of GFP blue excitation light pulses for GFP imaging $(200 \mathrm{~ms})$ were interspersed with $4.5 \mathrm{~s}$ of 625 or 740 $\mathrm{nm}$ light pulses at $10 \%$ and $100 \%$ of maximal LED power, respectively. To allow for spontaneous relaxation of activated PhyB following global activation of PhyB-PIF dimerization, the entire illumination and excitation scheme was paused for 30 min to prevent reactivation of PhyB-PIF dimerization by blue excitation light pulses.

For local activation of PhyB-PIF dimerization, after each GFP excitation, the activation ROI was illuminated with $2 \%$ $625 \mathrm{~nm}$ LED power for $500 \mathrm{~ms}$ followed by a $3.8 \mathrm{~s}$ exposure of the surrounding inactivation area with $100 \% 740 \mathrm{~nm}$ LED power. To locally dissociate PhyB-PIF dimers, a ROI was illuminated with $100 \% 740 \mathrm{~nm}$ LED power for $3.8 \mathrm{~s}$, after illuminating the remainder of the cell with $2 \% 625 \mathrm{~nm}$ LED power for $500 \mathrm{~ms}$ to keep it activated between excitation pulses. It is important to note that without far-red illumination between imaging excitations, PhyB will be activated by the light required to image eGFP.

For global activation of LOV-ePDZ dimerization, light pulses for tagRFP imaging $(50 \mathrm{~ms})$ were interspersed with $29 \mathrm{~s}$ of 470 $\mathrm{nm}$ light pulses at $10 \%$ maximal LED power. For local activation of LOV-ePDZ dimerization, the activation ROI was illuminated with $10 \% 470 \mathrm{~nm}$ LED power for $4.5 \mathrm{~s}$, after each excitation of tagRFP.

For orthogonal control optogenetic control of LOV-ePDZ and PhyB-PIF dimerization in the same cell, a light exposure scheme was preprogrammed on the digital mirror device to run independently of camera shutters and manually initiated to run synchronously with excitation light pulses and camera frames with the following effect: excitation light pulses for Venus imaging (514 bandpass filter set; $200 \mathrm{~ms}$ ) and mRFP imaging (mCherry filter set; $50 \mathrm{~ms}$ ), acquired at a $5 \mathrm{~s}$ interval, were interspersed with three different illumination regimes for sequential activation of PhyB-PIF and LOV-ePDZ dimerization. In the first regime, in which both systems remain inactive, cells were illuminated with $100 \% 740 \mathrm{~nm}$ LED power for $2.2 \mathrm{~s}$. In the second regime, which induced the dimerization of PhyBPIF but not of LOV-ePDZ, cells were illuminated with 50\% 625 $\mathrm{nm}$ LED power for $0.5 \mathrm{~s}$. In the third regime, which induced the dimerization of LOV-ePDZ, but not of PhyB-PIF, cells were illuminated with $50 \% 470 \mathrm{~nm}$ LED power for $0.5 \mathrm{~s}$ and subsequently illuminated with $100 \% 740 \mathrm{~nm}$ LED power for $2.2 \mathrm{~s}$.

Analysis of Endosome Distribution. Analyses of acquired images were performed with FIJI software and resulting graphs were plotted with Graphpad Prism 5. To quantify the effect of organelle repositioning in fixed cell experiments, organelle localization in cells of at least 2 coverslips were categorized as perinuclear enriched, evenly spread throughout the cytoplasm or clearly enriched at the cell periphery (see Figure 1). The number of cells $(n)$ is shown for each experiment. To quantify the efficiency of organelle repositioning in response to illumination with different wavelengths, we quantified the cumulative signal of thresholded (top $8 \%$ of signal) Phy-Rab11 measured along radial ROIs from the center to the periphery of the cell relative to the sum of all signal measured. The intercept of the resulting curve with $y=0.9$ indicated the radius of a circle surrounding $90 \%$ of all organelles.
To quantify peripheral organelle enrichments over time in live cell experiments, fluorescence intensity relative to the average of the first 10 imaging frames (baseline before induction of dimerization) was plotted as measured in background subtracted (rolling ball radius of $20 \mathrm{px}$ ) ROIs located as indicated. Further measures of organelle motility and repositioning (correlation index, tracking and displacement measures) were done as described previously. ${ }^{7,9}$

\section{ASSOCIATED CONTENT}

\section{S Supporting Information}

The Supporting Information is available free of charge on the ACS Publications website at DOI: 10.1021/acssynbio.6b00333.

Figures S1-S3 (PDF)

Movie S1 (AVI)

Movie S2 (AVI)

Movie S3 (AVI)

Movie S4 (AVI)

\section{AUTHOR INFORMATION}

\section{Corresponding Author}

*E-mail: 1.kapitein@uu.nl.

ORCID

Max Adrian: 0000-0002-4926-1258

Lukas C. Kapitein: 0000-0001-9418-6739

\section{Author Contributions}

M.A., W.N., R.I.H. and J.W. performed experiments. M.A. and W.N. analyzed and interpreted the data. M.A. and L.C.K. wrote the manuscript. L.C.K. supervised the study.

Notes

The authors declare no competing financial interest.

\section{ACKNOWLEDGMENTS}

This work is supported by The Netherlands Organisation for Scientific Research (NWO) (NWO-ALW-VIDI to L.C.K. and NWO-ALW-VENI to W.N), the Foundation for Fundamental Research on Matter (FOM), which is part of the NWO, and the European Research Council (ERC Starting Grant to L.C.K.).

\section{REFERENCES}

(1) van Bergeijk, P., Hoogenraad, C. C., and Kapitein, L. C. (2016) Right Time, Right Place: Probing the Functions of Organelle Positioning. Trends Cell Biol. 26, 121-34.

(2) Jongsma, M. L. M., Berlin, I., and Neefjes, J. (2015) On the move: organelle dynamics during mitosis. Trends Cell Biol. 25, 112-24.

(3) Prinz, W. A. (2014) Bridging the gap: membrane contact sites in signaling, metabolism, and organelle dynamics. J. Cell Biol. 205, 75969.

(4) Helle, S. C. J., Kanfer, G., Kolar, K., Lang, A., Michel, A. H., and Kornmann, B. (2013) Organization and function of membrane contact sites. Biochim. Biophys. Acta, Mol. Cell Res. 1833, 2526.

(5) Schrader, M., Godinho, L. F., Costello, J. L., and Islinger, M. (2015) The different facets of organelle interplay-an overview of organelle interactions. Front. Cell Dev. Biol. 3, 56.

(6) Rambold, A. S., Cohen, S., and Lippincott-Schwartz, J. (2015) Fatty acid trafficking in starved cells: Regulation by lipid droplet lipolysis, autophagy, and mitochondrial fusion dynamics. Dev. Cell 32, 678-692.

(7) Kapitein, L. C., Schlager, M. A., van der Zwan, W. A., Wulf, P. S., Keijzer, N., and Hoogenraad, C. C. (2010) Probing Intracellular Motor Protein Activity Using an Inducible Cargo Trafficking Assay. Biophys. J. 99, 2143-2152. 
(8) Duan, L., Che, D., Zhang, K., Ong, Q., Guo, S., and Cui, B. (2015) Optogenetic Control of Molecular Motors and Organelle Distributions in Cells. Chem. Biol. 22, 1-12.

(9) Bergeijk, P. V., Adrian, M., Hoogenraad, C. C., and Kapitein, L. C. (2015) Optogenetic control of organelle transport and positioning. Nature 518, 111-114.

(10) Harterink, M., van Bergeijk, P., Allier, C., de Haan, B., van den Heuvel, S., Hoogenraad, C. C., and Kapitein, L. C. (2016) Lightcontrolled intracellular transport in Caenorhabditis elegans. Curr. Biol. 26, R153-4.

(11) Strickland, D., Lin, Y., Wagner, E., Hope, C. M., Zayner, J., Antoniou, C., Sosnick, T. R., Weiss, E. L., and Glotzer, M. (2012) TULIPs: tunable, light-controlled interacting protein tags for cell biology. Nat. Methods 9, 379-84.

(12) Kennedy, M. J., Hughes, R. M., Peteya, L. A., Schwartz, J. W., Ehlers, M. D., and Tucker, C. L. (2010) Rapid blue-light-mediated induction of protein interactions in living cells. Nat. Methods 7, 973-5.

(13) Ballister, E. R., Ayloo, S., Chenoweth, D. M., Lampson, M. A., and Holzbaur, E. L. F. (2015) Optogenetic control of organelle transport using a photocaged chemical inducer of dimerization. Curr. Biol. 25, R407-R408.

(14) Guntas, G., Hallett, R. A., Zimmerman, S. P., Williams, T., Yumerefendi, H., Bear, J. E., and Kuhlman, B. (2015) Engineering an improved light-induced dimer (iLID) for controlling the localization and activity of signaling proteins. Proc. Natl. Acad. Sci. U. S. A. 112, $112-117$.

(15) Zimmerman, S. P., Hallett, R. A., Bourke, A. M., Bear, J. E., Kennedy, M. J., and Kuhlman, B. (2016) Tuning the Binding Affinities and Reversion Kinetics of a Light Inducible Dimer Allows Control of Transmembrane Protein Localization. Biochemistry 55, 5264-5271.

(16) Levskaya, A., Weiner, O. D., Lim, W. A., and Voigt, C. A. (2009) Spatiotemporal control of cell signalling using a light-switchable protein interaction. Nature 461, 997-1001.

(17) Ni, M., Tepperman, J. M., and Quail, P. H. (1999) Binding of phytochrome B to its nuclear signalling partner PIF3 is reversibly induced by light. Nature 400, 781-4.

(18) Shimizu-Sato, S., Huq, E., Tepperman, J. M., and Quail, P. H. (2002) A light-switchable gene promoter system. Nat. Biotechnol. 20, 1041-4.

(19) Beyer, H. M., Juillot, S., Herbst, K., Samodelov, S. L., Müller, K., Schamel, W. W., Römer, W., Schäfer, E., Nagy, F., Strähle, U., Weber, W., and Zurbriggen, M. D. (2015) Red Light-Regulated Reversible Nuclear Localization of Proteins in Mammalian Cells and Zebrafish. ACS Synth. Biol. 4, 951-958.

(20) Müller, K., Engesser, R., Metzger, S., Schulz, S., Kämpf, M. M., Busacker, M., Steinberg, T., Tomakidi, P., Ehrbar, M., Nagy, F., Timmer, J., Zubriggen, M. D., and Weber, W. (2013) A red/far-red light-responsive bi-stable toggle switch to control gene expression in mammalian cells. Nucleic Acids Res. 41, e77.

(21) Toettcher, J. E., Weiner, O. D., and Lim, W. A. (2013) Using optogenetics to interrogate the dynamic control of signal transmission by the ras/erk module. Cell 155, 1422-34.

(22) Pathak, G. P., Strickland, D., Vrana, J. D., and Tucker, C. L. (2014) Benchmarking of optical dimerizer systems. ACS Synth. Biol. 3, $832-8$.

(23) Müller, K., Engesser, R., Timmer, J., Zurbriggen, M. D., and Weber, W. (2014) Orthogonal optogenetic triple-gene control in Mammalian cells. ACS Synth. Biol. 3, 796-801.

(24) Müller, K., Engesser, R., Timmer, J., Nagy, F., Zurbriggen, M. D., and Weber, W. (2013) Synthesis of phycocyanobilin in mammalian cells. Chem. Commun. (Cambridge, U. K.) 49, 8970-2.

(25) Kaberniuk, A. A., Shemetov, A. A., and Verkhusha, V. V. (2016) A bacterial phytochrome-based optogenetic system controllable with near-infrared light. Nat. Methods 13, 591-597.

(26) Kapitein, L. C., Schlager, M., Kuijpers, M., Wulf, P. S., van Spronsen, M., MacKintosh, F. C., and Hoogenraad, C. C. (2010) Mixed microtubules steer dynein-driven cargo transport into dendrites. Curr. Biol. 20, 290-9. 